\title{
Unravelling body plan and axial evolution in the Bilateria with molecular phylogenetic markers
}

\author{
Jaume Baguñà, Pere Martinez, Jordi Paps and Marta Riutort
}

\section{SETTING THE PROBLEM}

The emergence of dramatic morphological differences (disparity) and the ensuing bewildering increase in the number of species (diversity) documented in the fossil record at key stages of animal and plant evolution have defied, and still defy, the explanatory powers of Darwin's theory of evolution by natural selection. Among the best examples that have captured the imagination of the layman and the interest of scores of scientists for I 50 years are the origins of land plants from aquatic green plants, of flowering plants from seed plants, of chordates from non-chordates and of tetrapod vertebrates from non-tetrapods; and the conquest of the land by amphibians; the emergence of endotherms from ectotherm animals; the recurrent invention of flight (e.g. in arthropods, birds and mammals) from non-flying ancestors; and the origin of aquatic mammals from fourlegged terrestrial ancestors.

Key morphological transitions pose a basic difficulty: reconstruction of ancestral traits of derived clades is problematic because of a lack of transitional forms in the fossil record and obscure homologies between 'ancestral' and derived groups. Lack of transitional forms, in other words gaps in the fossil record, brought into question one of the basic tenets of Darwin's theory, namely gradualism, as Darwin himself acknowledged. Since Darwin, however, and especially in the past 50 years, numerous examples that may reflect transitional stages between major groups of organisms have accumulated. Good examples are the numerous fossils that connect whales, sirenians, seals and sea lions with different lineages of terrestrial mammals, the converse transitional series from swimming tetrapods to land tetrapods, the many fossils showing the transition from dinosaurs to birds illuminating the origin and early functions of feathers and flight, and

Evolving Pathways: Key Themes in Evolutionary Developmental Biology, ed. Alessandro Minelli and Guiseppe Fusco. Published by Cambridge University Press. (C) Cambridge University Press 2008. 
those fossils illustrating the intermediate changes during the transition from aquatic green plants to land plants and from these to vascular plants.

Back in geological time, the last and potentially crippling example to the acceptance of the Darwinian theory is the advent of bilaterally symmetrical animals and its coincidence with the abrupt appearance of large-bodied skeletonised remains of most extant phyla. The event is usually referred as the Cambrian 'explosion'. A great deal has been written about it, namely the recent reviews by Budd (2003), Conway Morris (2006), Valentine (2004) and Marshall (2006), to which readers are referred. In the writings of Gould ( 1989 ) the Cambrian 'explosion' has been considered the pivotal event in animal evolution for which special mechanisms have been sought, e.g. in terms of macro-evolutionary events. However, because the Cambrian 'explosion' mainly refers to the 'explosion' of bilaterally symmetrical body plans, we will argue that an understanding of the origin of bilateral organisms is even more important than the so-called Cambrian 'explosion', as well as a necessary step to explain it.

\section{TRACKING DOWN THE EARLIEST EXTANT BILATERIANS: A}

SIMPLE OR A COMPLEX LAST COMMON ANCESTOR (LCA)?

By any standard, the appearance of bilateral organisms is the most thrilling success in animal evolution: 34 out of the 38 living phyla and over $99 \%$ of described living animal species are bilaterians, far more complex in structure and far more diverse in morphology and ecology than their radial forebears. A brief glimpse at any bilaterian organism, however simple, uncovers the main reasons for their evolutionary success: two oriented body axes and directed locomotion. The main or primary axis (antero-posterior, or A-P) distinguishes 'front' from 'back' of the body and is associated with the direction of locomotion, with the mouth, brain and sensory structures located at or near the anterior end, and the anus and other structures located at or near the posterior end. The second axis (dorso-ventral or D-V), orthogonal to the first, identifies the 'top' from the 'bottom' of the body, the latter usually related to locomotion, while the 'top' or dorsal bears sensory and defensive structures to avoid predation. Oriented locomotion was the key to the colonisation by the pre-Cambrian benthos and thereafter the plankton, and aided by the development of sensory structures and feeding organs at the anterior/ventral end that increased predatory and escape capabilities. Another key feature of bilaterians is the presence of a third embryonic layer, the mesoderm, between the ectoderm and endoderm. In combination with either the ecto- or endoderm, the mesoderm provides an extraordinary 
variety of new tissues and organs not seen in any radial organism. Finally, other features often considered to be present in the first bilaterians are a true brain, a through-gut, excretory system, body cavities (coelom), segments, and even appendages and simple hearts and eyes (Table I 2.I).

Current views suggest that the bilaterians arose from ancestors that were radially symmetric instead of bilateral and, therefore, had a single body axis (the oral-aboral, or $\mathrm{O}-\mathrm{AB}$ ) and no mesoderm (hence diploblastic). In addition, they had a decentralised nerve net and a blind gut. These features are maintained by the extant members of the phylum Cnidaria (corals, sea anemones, hydras and jellyfish) and Ctenophora (comb jellies). However, whenever a hypothetical early bilaterian with the first, second or third set of apomorphic (derived) characters (Table I2.I) is compared with a radial organism bearing none of them, and from which it is assumed to originate, one is left wondering how this actually took place.

Since Haeckel's Gastraea, scores of theories have tried to answer this key evolutionary question (see Willmer 1990, for a historical review, and

Table I2.I Character states of the main morphological and developmental components at the dawn of bilaterians.

The simple Urbilateria scenario assumes a structurally simple organism. The alternative complex Urbilateria scenario considers that most morphological and developmental components of extant bilaterians were also functionally conserved in the bilaterian ancestor.

\begin{tabular}{lcc}
\hline \hline $\begin{array}{l}\text { Developmental and morphological } \\
\text { characters }\end{array}$ & Simple Urbilaterian & Complex Urbilaterian \\
\hline I - A-P axis & Present & Present \\
2 - D-V axis & Present & Present \\
3- Mesoderm & Present & Present \\
4- Nervous system & Present (slightly & Present (centralised; \\
& centralised) & CNS) \\
5- Hox cluster & Basic (3-4 genes) & Expanded (7-9 genes) \\
6- Brain & Clumps of cells & Present (true brain) \\
7 - Gut & Blind gut & Through-gut \\
8- Excretory system & Absent & Present \\
9- microRNAs & ? (few) & ? (some) \\
I0 - Body cavities (coelom) & Absent & Present \\
I - Segmentation & Absent & Present \\
I2 - Heart & Absent & Present \\
I3 - Appendages & Absent & Present? \\
I4 - Body size & Small & Large \\
I5 - Life cycle & Direct & Indirect (+larvae) \\
\hline \hline
\end{tabular}


Holland 2003 , for details on the evolution of the nervous system). In a first major set of hypotheses, ancestral bilaterian traits such as body axes and mesoderm appeared concurrently with advanced characters such as coelom and segments. Hence, non-segmented, non-coelomate cnidarians with blind guts, either under larval or adult appearance, were directly transformed to coelomate segmented bilaterians, bearing through-guts and complex nervous systems (Archicoelomate Theories) (for a recent critical update, see Holland 2003). A second major set of hypotheses (see SalviniPlawen 1978 for a thorough review) featured a more gradual scenario from sexually reproducing, bottom-pelagic organisms (protoplanula or archiplanula), akin to present cnidarian planula larva, already exhibiting bilateral symmetry. From such organisms originated the cnidarian polyps, which settled onto the substratum, as well as the early bilaterians which resembled present day acoel and nemertodermatid flatworms (Planulaacoeloid Theory). Accordingly, the first bilaterians were non-segmented, non-coelomate (acoelomate) organisms with a blind gut from which pseudocoelomate and coelomate, segmented and non-segmented protostomes and deuterostomes evolved.

The phylogenetic consequences of these conflicting scenarios, in terms of character changes necessary between ancestors and descendants, are very different. Under the archicoelomate scenario, the number of coincident characters clumping at the Last Common Ancestor (LCA) node of the bilaterians is large. This makes it difficult to place them into any temporal order along the stem leading to the LCA (Figure I 2.IA). Also, it implies either a large number of extinctions of intermediary taxa and, consequently, major gaps in our knowledge, or a wholesale correlated transformation from one life form (radial) to another (bilateral). Under this hypothesis, the LCA appears as a rather complex organism (dubbed complex Urbilateria; Kimmel 1996). In contrast, the planuloid-acoeloid scenario posits a reduced number of characters at the stem leading to the LCA (Figure I 2.IB), and features fewer and simpler stem ancestors and a simple LCA. Under both scenarios, however, phylogenetic advances may discover extinct (or hopefully extant) clades that break coincident character changes at the stem. The intercalation of these new clades will distribute inferred character changes across a series of branches instead to having them distributed solely at the LCA node (Donoghue 2005, Butterfield 2006).

In the I990s, molecular phylogenies based on sequences of the ribosomal gene $18 \mathrm{~S}$ and the Hox gene clusters bolstered the Archicoelomate scenario (and the complex Urbilateria). Both sets of data split the Bilateria into three superclades, the classical Deuterostomia and the protostomes divided into 
(A)

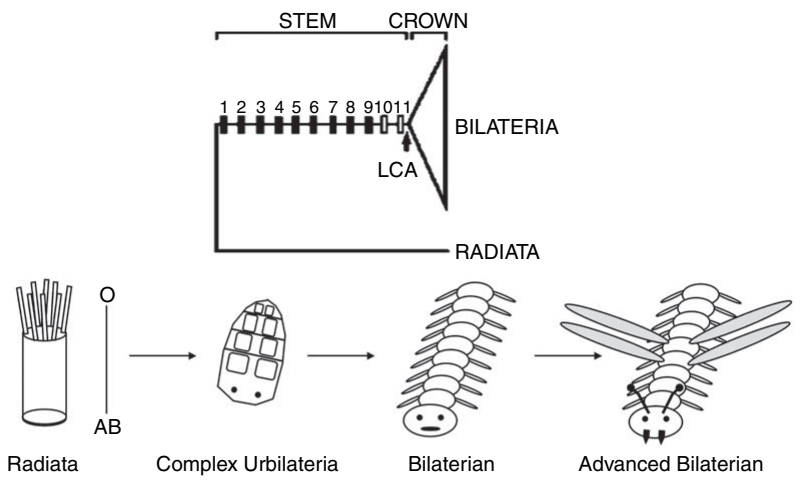

(B)

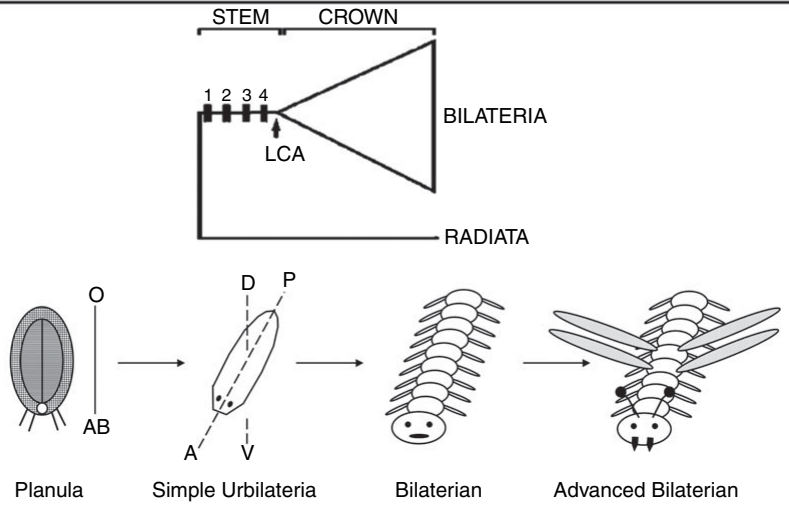

Figure I2.I Conflicting phylogenies and scenarios on the nature and origin of the Last Common Ancestor (LCA) of the Bilateria, also featuring the extent of stem and crown groups. A, The complex Urbilateria scenario features a large, complex ancestor bearing most characters of present-day bilaterians (characters $\mathrm{I}-9$, and eventually characters $\mathrm{IO}-\mathrm{I} \mathrm{I}$ in Table I 2.I). This ancestor originated from either an adult (polyp) or a larval radial cnidarian (archicoelomate theory, originally proposed by Sedgwick i 884). From this LCA evolved the more complex protostomes and deuterostomes. Note that all characters leading to the LCA are clumped at the stem. The large triangle indicates the diversification of crown bilaterians and its short height shows that its rate was fast (Cambrian 'explosion'?). B, The simple Urbilateria scenario features a small, simple LCA, similar to present-day acoelomorph flatworms, bearing a reduced set of characters ( $\mathrm{I}-4$ of Table I 2.I ) of extant bilaterians. This ancestor originated from radial planuloid ancestors similar to the planula larva of extant cnidarians (planuloid-acoeloid theory; for main references see Salvini-Plawen 1978 and Willmer 1990). From this ancestor evolved more complex bilaterians to be followed by the most advanced protostomes and deuterostomes. Note that the number of characters leading to the LCA are few, that time of diversification of crown bilaterians was longer and its rate slower than in the alternative scenario. A: anterior; $\mathrm{AB}$ : aboral; D: dorsal; O: oral; P: posterior; V: ventral. 
Ecdysozoa (Aguinaldo et al. 1997) and Lophotrochozoa (Halanych et al. 1995). The Ecdysozoa clustered several pseudocoelomate groups with arthropods, while the Lophotrochozoa joined most acoelomates (e.g. Platyhelminthes) to coelomate spiralians and lophophorates. Acoelomates and pseudocoelomates were displaced to more derived positions inside the tree and, therefore, had to originate by morphological simplification from complex coelomate segmented ancestors. Moreover, the amazing conservation of the genetic toolkit across the Bilateria, together with the apparently homologous expression of key developmental genes (e.g. segmentation and nervous system genes) in disparate bilaterian clades (annelids, insects, vertebrates; De Robertis and Sasai 1996, P. W. H. Holland 1998, L. Z. Holland 2000) were taken as evidence for the existence of similar developmental programs and their ensuing morphological characters in the Urbilateria ancestor. Finally, the lack of resolution of branching phyla within the three superclades gave support to the Cambrian 'explosion' as a real, sudden, cladogenetic event. In summary, hopes of finding extant 'intermediates' in the bilaterian stem lineage were considered doomed (Adoutte et al. 1999), the gradist interpretation of early bilaterian evolution dismissed, and the complex Urbilateria enthroned (Carroll et al. 200I).

\section{THE ACOELOMORPHA, A LIKELY CANDIDATE FOR THE EARLIEST BRANCHING EXTANT BILATERIANS}

Whereas the splitting of the Bilateria into the three superclades was corroborated by further data, other tenets of the new phylogeny proved unfounded. First, most new phylogenies were heavily pruned, leaving out several 'minor' phyla, namely 'basal' ecdysozoans and lophotrochozoans, to which most pseudocoelomates and acoelomates belong (Jenner 2000). Phylogenies of both superclades which include these 'minor' phyla (e.g. Gastrotricha, Gnathostomula, Rotifera, Priapula, Kynorhyncha, Rhabditophora, Chaetognatha) show them to branch at or near the base of the tree (Glenner et al. 2004, Peterson et al. 2005, Mallatt and Giribet 2006). That makes untenable the proposal that most pseudocoelomate and acoelomate groups are secondarily derived from more complex ancestors. Second, similar expression patterns of key developmental genes (De Robertis and Sasai 1996, P. W. H. Holland I998, L. Z. Holland 2000), taken as evidence of deep 'functional' homologies across the Bilateria, were found to be rather variable and it remained unclear whether they refer to cell-type specification or morphogenetic processes (Erwin and Davidson 2002, Nielsen and Martinez 2003). Moreover, they were not coded as characters and tested 
in a wide phylogenetic-cladistic analysis (Hübner 2006). Finally, molecular trees of the phylum Platyhelminthes showed it to be polyphyletic (Ruiz-Trillo et al. I 999, 2002). Indeed, the platyhelminth orders Acoela and Nemertodermatida branched at the base of the bilaterians while the rest of the phylum (Catenulida + Rhabditophora) fell at variable positions within the Lophotrochozoa (Ruiz-Trillo et al. 1999, Jondelius et al. 2002, Baguñà and Riutort 2004). Such a basal position was corroborated from sequences of other nuclear genes (Ruiz-Trillo et al. 2002, Telford et al. 2003) including Hox genes (Cook et al. 2004), mitochondrial genes (Ruiz-Trillo et al. 2004), and from the first microRNA (miRNA) gene tested in a large set of Metazoans and found absent in diploblasts and acoels (Pasquinelli et al. 2003) (see below).

The proposal of Acoelomorpha (Acoela + Nemertodermatida) as the extant earliest branching bilaterians divides the Bilateria into two inclusive groups: a broad Bilateria including acoelomorphs, and a more derived Bilateria, named Eubilateria (Baguñà and Riutort 2004) or Nephrozoa (Jondelius et al. 2002), excluding this clade. The new phylogenetic proposal is fairly close to the planuloid-acoeloid scenario of Figure I 2. I B. It puts back in time and reduces the number of character states leading to the LCA of bilaterians, and suggests that the LCA was small, acoelomate, unsegmented and a direct developer. However, it is very important to stress that Acoelomorpha, and acoels in particular, are by no means equivalent to the bilaterian LCA. They bear, among others, several autapomorphic characters (e.g. duet-spiral cleavage, an interconnecting ciliary rootlet system and bent cilia at terminal ends) which makes them a rather specialised group (Ax 1996).

NEW MOLECULAR DATA: NUCLEAR GENES, HOX CLUSTER GENES, EST COLLECTIONS, AND MICRORNA SETS

\section{Nuclear genes}

The $18 \mathrm{~S}$ and $28 \mathrm{~S}$ ribosomal genes and the myosin heavy chain gene, together with io new nuclear genes from a large taxon sample $(63$ species belonging to is phyla) have been used to further test the basal position of acoelomorphs. Combined $18 \mathrm{~S}+28 \mathrm{~S}$ trees and concatenated datasets totalling I 3 genes gave similar results (Figure I 2.2 for the I 3 gene dataset; J. Paps, J. Baguñà and M. Riutort, unpublished data). Acoels and nemertodermatids branch in sequence with high support at the base of the bilaterians. Further, the three superclades are well resolved and some interesting internal clusterings suggested (e.g. Priapulida, Kynorhyncha and 


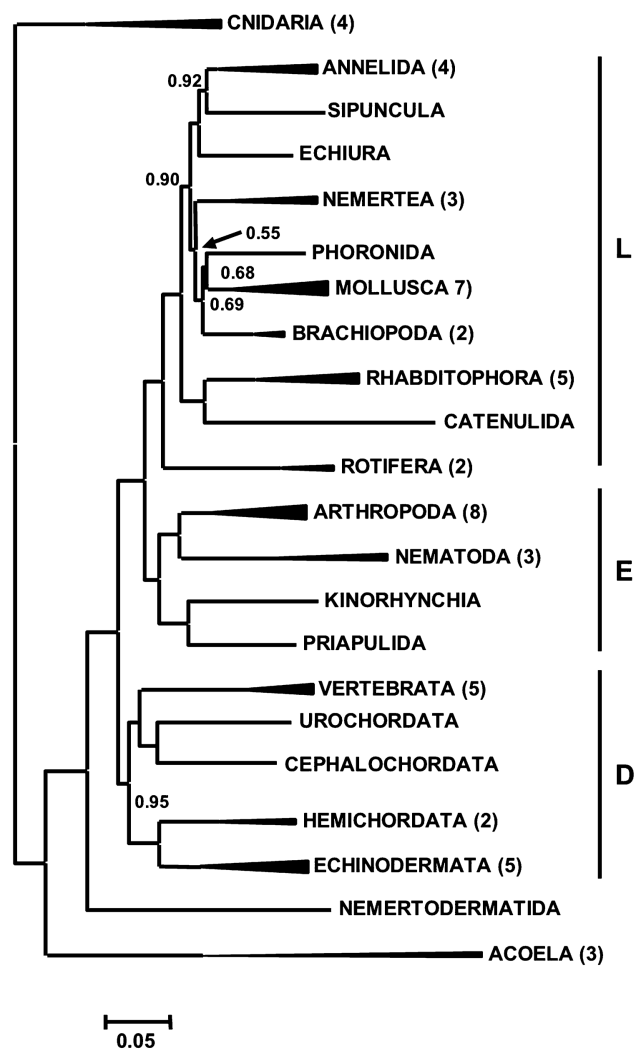

Figure I 2.2 Phylogeny of bilaterians determined by Bayesian inference (MRBAYES using a GTR model and gamma distribution) from concatenated sequences of I 3 genes ( 18 and $28 \mathrm{~S}$ rDNA and I I nuclear genes, 8446 nucleotides) from 63 species belonging to i 9 metazoan phyla. All nodes show a maximum BPP (Bayesian Posterior Probability, obtained from I 000.000 replicates analysis) value of I.00, except those at some specific nodes. In brackets, number of species per phylum, except those with single representatives. D: Deuterostomia; E: Ecdysozoa: L: Lophotrochozoa. Scale bar indicates the number of substitutions per position (from J. Paps, J. Baguñà and M. Riutort, unpublished data).

Nematoda at the base of the Ecdysozoa, and Rotifera and Platyhelminthes (Catenulida + Rhabditophora) at the base of the Lophotrochozoa).

\section{EST (Expressed Sequence Tags) collections}

Complete genomes of several model systems (e.g. yeasts, Drosophila, Caenorhabditis, Mus, Homo) have been used to gather large numbers (> 100) of 
homologous genes to examine the basic tenets of the new molecular phylogeny. Surprisingly, the first phylogenies failed to recover the superclade Ecdysozoa (Blair et al. 2002, Dopazo et al. 2004). However, while a large number of genes reduces the impact of stochastic errors of single-gene phylogenies, it does not deal with systematic errors. Such errors plagued early genome-derived phylogenies because sampling was poor (four to six species) and species had high/very high rates of nucleotide substitution (Jeffroy et al. 2006). To overcome these problems, a large number of both genes and species was used, and the new animal phylogeny and the clade Ecdysozoa were recovered again (Philippe et al. 2005). Rather than waiting for complete genomes of taxa from each phylum, the most convenient and less expensive approach is to sequence a small number of Expressed Sequence Tags (1000-5000 ESTs per species) from as many taxa as possible (Philippe and Telford 2006).

EST collections from 60 metazoan species belonging to i 3 phyla, and an EST collection from the acoel Convoluta pulchra, have been used to test the basal position of acoels (H. Philippe, J. Baguñà, M. Riutort and P. Martinez, unpublished results). To avoid long-branch problems caused by fastevolving clades (Convoluta pulchra among them), we introduced a siteheterogeneous mixture model (CAT; Lartillot et al. 2007) instead of standard, site-homogeneous models. Preliminary trees run under PhyloBayes (I I 000 amino acid positions) resolve the bilaterians into the three big superclades, with sponges and cnidarians branching earlier, Platyhelminthes within the Lophotrochozoa, and acoels in an unstable position as a basal clade to bilaterians, protostomes or deuterostomes. Although the final position of acoels is unresolved (probably because Convoluta pulchra is a very fast-evolving species), it confirms clearly that acoels are not members of the Platyhelminthes.

\section{Hox cluster genes}

The Hox and ParaHox genes code for transcription factors that regulate A-P patterning in many bilaterian phyla. Most bilaterians have a Hox cluster comprising at least seven to eight distinct genes, or paralogy groups (PGs), and a ParaHox set bearing three genes usually not clustered. Therefore, finding a full set of Hox cluster genes in acoelomorphs would confirm they are not basal bilaterians; conversely, finding a reduced gene set, intermediate between those of cnidarians and bilaterians, would support their position as early branching bilaterians. 
Hox and ParaHox genes have been isolated and analysed from five species of acoels and a single nemertodermatid (Cook et al. 2004, Jiménez-Guri et al. 2006, M. Q. Martindale, personal communication; P. Martinez and J. Baguñà, unpublished data). All acoels examined have a reduced complement of Hox genes: one anterior gene (PGi; an additional anterior gene exists in Convoluta pulchra; P. Martinez and J. Baguñà, unpublished data), one central gene (G4-5; Cook et al. 2004), and one posterior ( $\mathrm{PG}_{9-\mathrm{IO}}$; a second posterior gene is present in Paratomella rubra; Cook et al. 2004), and one posterior ParaHox gene $(C d x)$. The nemertodermatid Nemertoderma westbladi bears two central Hox genes (PG4-5 and PG6-8) and one posterior (PG9-10), and two ParaHox: an Xlox-PG3 and a $C d x$ (Jiménez-Guri et al. 2006). In summary, assuming that anterior and posterior additional Hox genes are species-specific duplications, acoelomorphs do have one anterior, one or two central, and one posterior Hox genes, and one representative each of the Xlox-PG 3 an $C d x$ ParaHox genes.

If a simple Hox gene cluster is substantiated in other acoelomorphs and found (or not) to be structurally collinear (E. Moreno, J. Baguñà and P. Martínez, work in progress) it might represent a simple Hox cluster intermediate between the simpler set of Hox/ParaHox genes in cnidarians and the expanded set (at least 7/8 PGs) of most bilaterians. Recent genome-wide analyses of two cnidarians (Nematostella vectensis and Hydra magnipapillata; Chourrout et al. 2006, Kamm et al. 2006) found anterior-like and extremely divergent 'posterior'-like Hox genes, no representatives of central genes, and a cluster of anterior and central/posterior ParaHox. This contradicts early claims of a ProtoHox cluster of four genes and a ParaHox cluster of three genes prior to cnidarian branching from which two Hox and one ParaHox were subsequently lost in the lineage leading to cnidarians (Brooke et al. i 998, Finnerty and Martindale i 999).

\section{MicroRNA (miRNA) sets}

MicroRNAs (miRNAs) are non-coding RNAs that control gene expression by decreasing the stability of translation of target mRNAs (reviewed by Wienholds and Plasterk 2005). MicroRNAs and their mRNA targets are usually expressed in mutually exclusive domains; in other words, repression of mRNAs in cell types where the miRNA is expressed suggests that miRNAs stabilises and confers robustness to cell differentiation (Stark et al. 2005). From this, it follows that the diversity of miRNAs might be correlated with the number of cell types and, hence, with biological complexity, both features having steadily increased along animal evolution. 


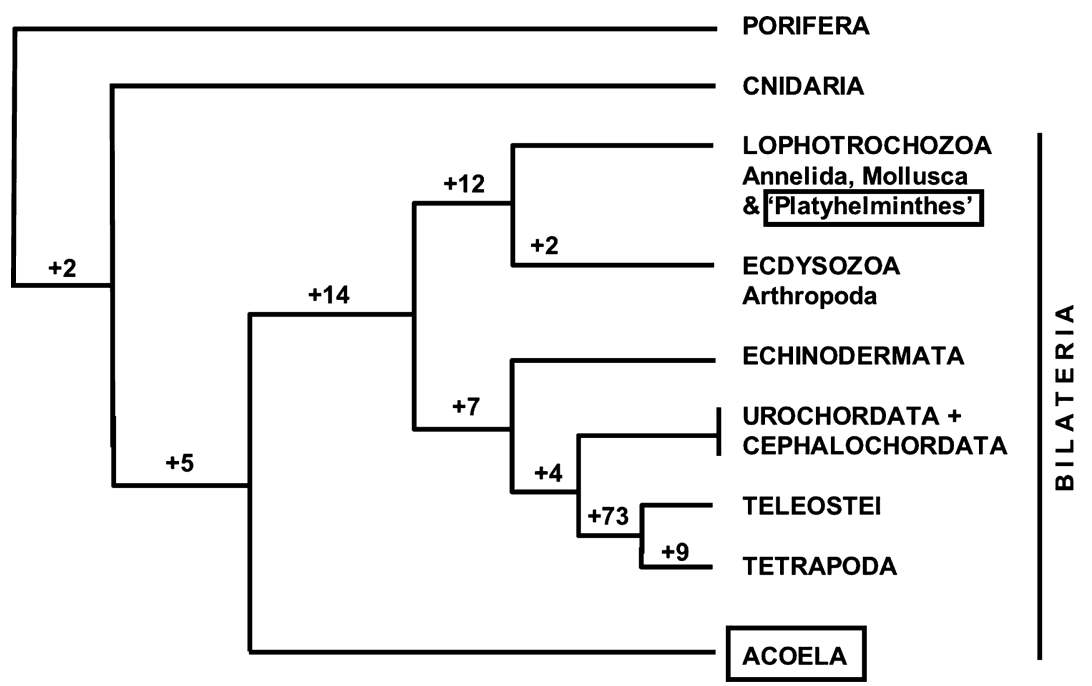

Figure I 2.3 An abbreviated phylogenetic tree depicting some metazoan clades with, above the nodes, the number of new miRNAs appearing at each cladogenetic event. The number of different miRNAs in Acoela is low (7 miRNAs) whereas that of 'Platyhelminthes' (Catenulida + Rhabditophora) is similar ( 33 miRNAs) to those of other lophotrochozoans like annelids and molluscs. This supports previous work suggesting the polyphyly of Platyhelminthes and the basal position of Acoelomorpha (Ruiz-Trillo et al. 1999, 2002). Redrawn in a very modified form from Sempere et al. 2006.

Recently, it has been reported that the number of different miRNAs roughly correlates with both the hierarchy of metazoan relationships and with the origination of metazoan morphological innovations through geological time (Sempere et al. 2006). The phylogenetic history (presence/ absence) of 243 human and 70 fruit fly non-paralogous miRNAs was traced along a wide range of taxa from sponges to humans using Northern blots. Twenty-one miRNAs were found common to protostomes and deuterostomes (Figure I 2.3) of which none is present in sponges and just two in cnidarians. Protostomes had $\mathrm{I} 2$ additional specific miRNAs and deuterostomes seven. Platyhelminthes, represented by a marine polyclad, had almost all protostome miRNAs excluding the two ecdysozoan-specific miRNAs so far detected, confirming that they are lophotrochozoan protostomes.

If acoels are early-branching bilaterians, they should bear a reduced subset of the $2 \mathrm{I}$ miRNAs conserved across protostomes and deuterostomes. Consistently, only six miRNAs were found in the acoel Childia sp. (Sempere et al. 2006). Additional species of Platyhelminthes (including 
parasitic species) have most protostome-specific miRNAs as well as those shared by protostomes and deuterostomes (L. F. Sempere, P. Martinez, J. Baguñà and K. J. Peterson, unpublished data). Instead, a second acoel examined, Symsagittifera roscoffensis, has the same six miRNAs as Childia sp. Again, these data strongly support the idea that acoels are early-branching bilaterians and not members of the Platyhelminthes.

GENE EXPRESSION AND AXIAL HOMOLOGIES BETWEEN

CNIDARIANS AND BILATERIANS

A major breakthrough in biology during the second half of the twentieth century has been the demonstration that, while animal phyla are morphologically very disparate, they are fundamentally similar genetically. While the genetic composition of extinct taxa (e.g. the LCA of bilaterians) cannot be directly determined, we can use the phylogenetic distribution of developmental genes in extant species to infer the 'genetic toolkit' of the bilaterian LCA. Within the framework of the new molecular phylogeny (Figure I2.IA), the bilaterian LCA is seen as endowed with scores of genes controlling, for example, body axiality, coelom formation and segmentation, photoreception, circulation and body appendages (Carroll et al. 200 I). Such a constellation of genes had to be assembled at the dawn of the Bilateria from radial ancestors not bearing them.

The way we look at the origin of bilaterality changed recently when it was found that the morphologically simple and symmetrically 'radial' anthozoan cnidarians possess, besides genes involved in A-P polarity (Hox/ParaHox, otx, ems, gsc), gastrulation (twist [twi], snail [sna], brachyury $[$ Bra], forkhead $[\mathrm{fkb}]$ ), endodermal (GATA) and germ-cell (nanos [nos], vasa [vas]) specification, orthologues to bilaterian gene families previously thought to be absent in 'radial' organisms. Prominent among them are genes involved in mesoderm specification (Nk2, mef2, MyoD), D-V axial polarity (Wnt-3-catenin, dpp/bmp; Chordin/noggin [chd/nog], Gsh/ind, $M s h$, vnd), nerve tissue and sensory-organ formation (Notch/Delta $[N / D l]$, Achete/Scute $\left[A c / S_{c}\right]$, Netrin, Pax 3$)$ as well as in other cell signalling pathways (hedgehog $[h b])$, Receptor tyrosine kinases (Egfr, Fgfr) and Jak/Stat (for specific references, see Hayward et al. 2002, Finnerty et al. 2004, Martindale et al. 2004, Extavour et al. 2005, Martindale 2005, Matus et al. 2006, Rentzsch et al. 2006). The presence and expression in cnidarians of many of the genes involved in $\mathrm{D}-\mathrm{V}$ patterning in bilaterians matched ideas (going back to Stephenson 1926, and held by Hyman I 95 I and Salvini-Plawen I978) of a second or directive axis in cnidarians 
(namely in anthozoans), perpendicular to the oral-aboral (O-AB) axis (Finnerty et al. 2004). Therefore, both cnidarians and bilaterians evolved from an ancestor already bilateral, putting the origin of the bilaterian LCA even further back in time.

Figure $\mathrm{I} 2.4$ summarises in a simplified form the A-P and D-V expression of selected developmental genes in cnidarians and bilaterians (for specific details see references above). Despite highly dynamic expressions, some A-P and D-V genes in cnidarians have patterns comparable to those of bilaterians. This seems so for gastrulation or 'posterior' genes such as $W n t, b r a$, sna, twi, fkh, for 'endodermal' or 'mesoendodermal' genes such as GATA, for 'mesodermal' genes like $N K_{2}$, mef2 and $M y o D$, and for germ-cell genes as nos and vas. However, the expression of key A-P genes such as Hox/ParaHox, emx , otx, Nkx2.5, and especially of key D-V genes such as $d p p / b m p$ and $c h d / n o g$, throws doubt on the existence of simple relationships between the $\mathrm{A}-\mathrm{P}$ and $\mathrm{D}-\mathrm{V}$ axes of bilaterians and the $\mathrm{O}-\mathrm{AB}$ and directive axes of cnidarians, respectively (Kamm et al. 2006, Chourrout et al. 2006, de Jong et al. 2006). Patterns of expression of A-P genes differ dramatically between different species and those of D-V genes are complex and overlapping (de Jong et al. 2006). In particular, the bilaterian antagonist factors $d p p / s o g$ (or $b m p / C h d$ in chordates) in Nematostella show asymmetric expression along the directive axis but, unexpectedly, also along the $\mathrm{O}-\mathrm{AB}$ axis (Rentzsch et al. 2006).

A particularly vexing old problem, which may hold the key to axial homologies, is the correspondence between the $\mathrm{O}-\mathrm{AB}$ axis of planula larva and polyp, and between these and the A-P axis of bilaterians. Planula larvae swim with the aboral or apical poles in front and the oral (bearing sometimes a transitory mouth) poles trailing. It is currently assumed that the aboral/oral (AB-O) axis in a planula corresponds to the A-P axis of bilaterians, and taking into account its directed locomotion, then $\mathrm{AB}=\mathrm{A}$ and $\mathrm{O}=\mathrm{P}$. After settling with the anterior pole, the larva transforms into a polyp having the oral end up and the aboral end at the bottom. If axiality between planula and polyp is conserved, the oral (mouth) of the polyp would correspond to the P pole of bilaterians while the aboral (basal disk and foot) would correspond to the A pole. This interpretation is backed by traditional morphological arguments and by the striking similarities between the oral region in cnidarians and the organiser region of chordates and other gastrulation sites of bilaterians which corresponds to either the posterior or ventral pole of modern-day bilaterians (Arendt et al. 200I, Technau 200I). Alternatively, the oral pole of the polyp may correspond to the bilaterian anterior pole (Martindale 2005). This would entail, however, the inversion of the A-P axis between planula and polyp. 


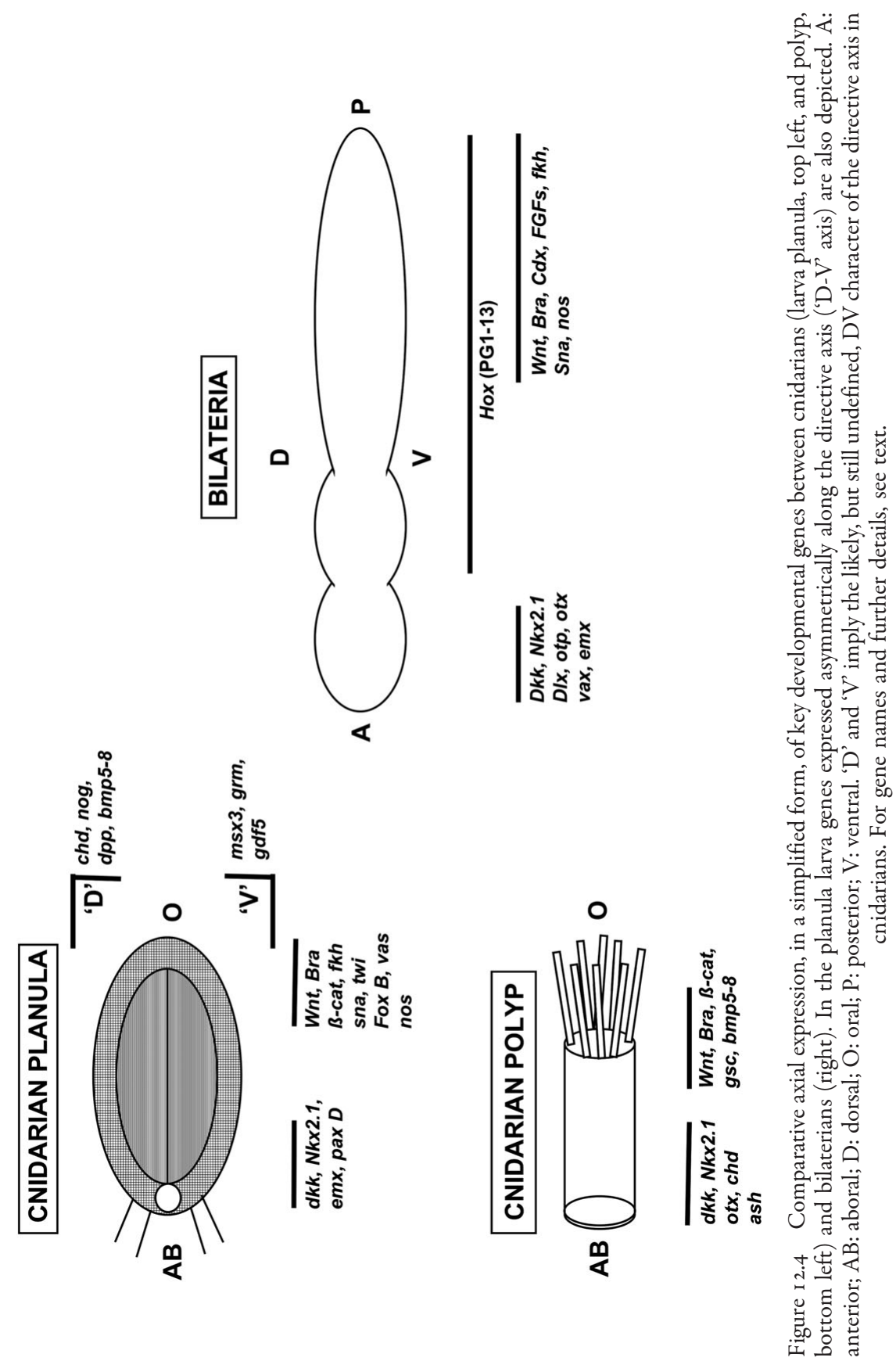


Gene expression in planula larvae (Figure I 2.4) does not provide a definitive answer, but gives interesting clues. The best come from sets of genes in the oral region of both planula and polyp. Wnt, $\beta$-cat, Bra, sna, twi, fkh, vas and nos are expressed in the posterior (oral) half of the planula larva and (some) in the hypostome area (oral pole) of the polyp. In bilaterians, such genes are expressed in posterior regions (including the posterior endoderm and germ cells) of the embryo and are involved in gastrulation and axial polarity. A second group of genes, Dickkopf $(D k k)$ and $N k x 2 . I$, are expressed in the anterior (aboral) half of the planula and in the peduncle and basal disk (aboral pole) of the polyp. Dkk is particularly interesting because it antagonises the $W n t$ signalling pathway in both cnidarians and bilaterians. Activation of $W n t$ signalling in bilaterians enlarges posterior structures and inhibits anterior structures; in cnidarians, it results in extra heads and tentacles (Guder et al. 2006). Conversely, depletion of Wnt activity in bilaterians expands anterior structures, whereas in cnidarians it gives rise to extra feet and basal discs. In vertebrates, $D k k_{I}$ is expressed in anterior regions and, when ectopically expressed, induces secondary heads (Glinka et al. 1997). In cnidarians, Dkk is expressed at the aboral end in the planula and polyp (Lee et al. 2006) Wnt is expressed at the oral end, and when $D k k$ is depleted, oral structures are expanded (Guder et al. 2006). If $W n t$ is considered a posterior marker in bilaterians and its antagonist $D k k$ an anterior marker, their expression in cnidarians and the results of over expression/inhibition suggest that the aboral end of the planula (= foot of polyp) is homologous to the anterior region of bilaterians (Meinhardt 2002), whereas the oral end of a planula (= hypostome of polyp) is homologous to the posterior region of bilaterians. Under this scenario, the postulated inversion of axial polarity between planula and polyp is neither necessary nor tenable.

THE PLANULA - ACOELOID THEORY REVISITED WITH A CRITIQUE

TO AMPHISTOMIC SCENARIOS OF BILATERIAN EVOLUTION

New molecular phylogenies (Figure I 2.2), new data on Hox/ParaHox and microRNA sets confirming the acoelomorphs as earliest extant branching bilaterians (Figure 12.3), the finding that all animal phyla (sponges included; Nichols et al. 2006) share a complex 'genetic toolkit', the evidence for axial homologies in gene expression between cnidarians and bilaterians (Figure I 2.4), and the evidence that cnidarians are bilateral in origin, all converge to an older LCA for bilaterians (Figure I2.5), better named the CBA (Cnidarian-Bilaterian Ancestor). In turn this resembled more 


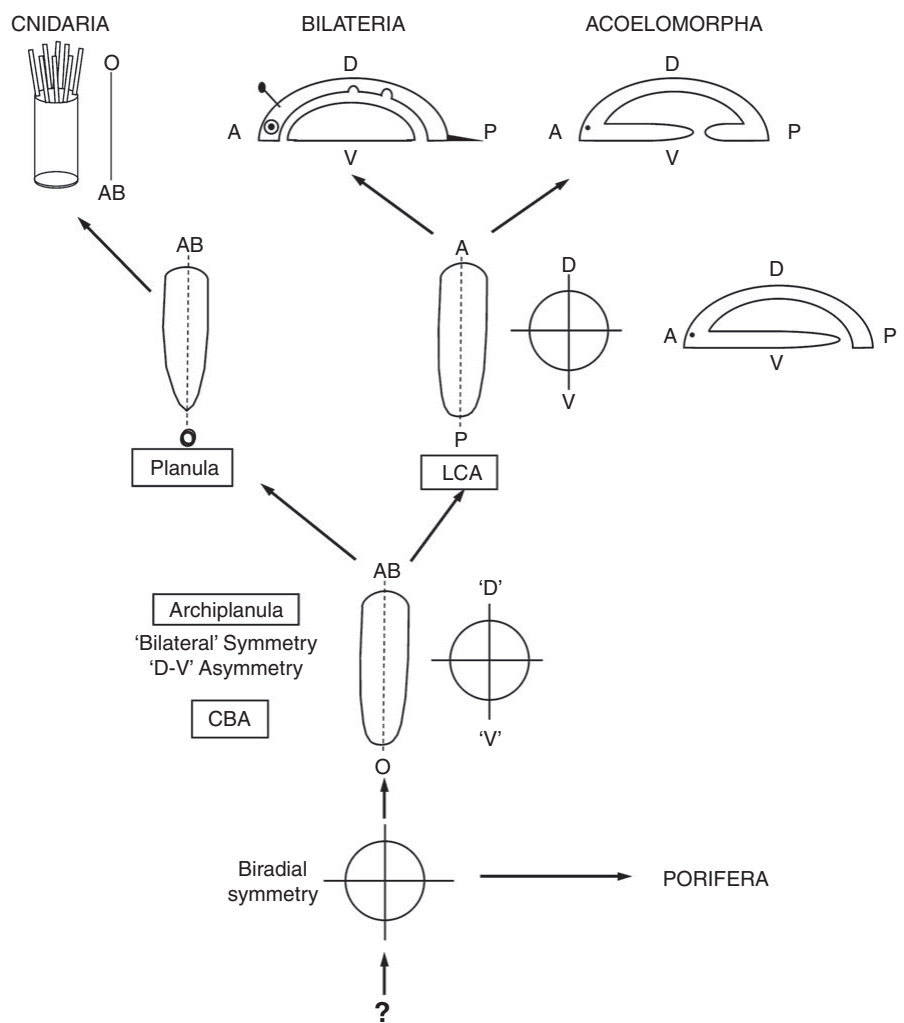

Figure I 2.5 Phylogenetic hypothesis for lower Metazoa, from biradial ancestors (crosssection) up to the archiplanula or CBA (Cnidarian-Bilaterian Ancestor) with loose 'bilateral' symmetry and 'dorsoventral' ('D-V') asymmetry and from the latter to both extant cnidarians via planula forms and to the Last Common Ancestor (LCA) of bilaterians. The LCA showed defined antero-posterior (A-P) and dorso-ventral (D-V) axes and from them derived both the acoelomorphs (right) and the rest of the bilaterians (centre). Drawn from concepts, ideas and phylogenetic schemes of Salvini-Plawen (1978), Willmer (1990), Baguñà and Riutort (2004) and Martindale (2005). For further details, see text.

closely the ancestor envisaged in the Planula-Acoeloid Theory, with an axial and bilateral, bentho-pelagic sexual archiplanula with directed locomotion, anterior sensory pole, posterior mouth and a rudimentary gut. From this ancestor, both cnidarians and 'true' bilaterals emerged. This scenario has affinities with the early ideas of Metchnikoff, further elaborated by Hyman, Beklemishev, Ivanov and later on by Salvini-Plawen (I978), to which readers are referred. 
Leaving aside sponges and placozoans, the primitive mode of feeding in metazoans appears to be grazing in and on the benthos, feeding upon organisms smaller than themselves such as bacteria, algae and other animals (Peterson et al. 2005). In other words, suspension feeding or active pelagic feeding, as in extant cnidarian polyps and ctenophores, was unlikely to be primitive. In both groups, it could only have occurred after the evolution of cnidoblasts (cnidarians) and colloblasts (ctenophores) which are no older than the Cambrian. This was concurrent with the appearance of appropriate food sources, namely mesozooplankton (Peterson 2005). Earliest cnidarians were probably small benthic grazers or burrowers with a main $\mathrm{A}-\mathrm{P}$ axis (equivalent to $\mathrm{AB}-\mathrm{O}$ ), the oral end (mouth/anus) at the rear, and a cryptic D-V axis. Once planulas of stem cnidarians developed a rudimentary pair of tentacles with primitive cnidocysts, and settled with the anterior pole to the substrate, ancestral archipolyps emerged ready to penetrate into the vacant ecological niche of sessile predators (Salvini-Plawen I 978).

Another group of bentho-pelagic sexual archiplanulas gave rise to stem bilaterians (Figure I 2.5). Given that A-P and 'D-V' axes were already in place, key apomorphies leading to the LCA were the reinforcement of the $\mathrm{D}-\mathrm{V}$ axis, probably helped by the appearance or 'segregation' of mesoderm from endomesoderm, and concentration at the anterior end of clumps of nerve cells to form a first primitive brain. A further or concurrent important development included the shift of the blastopore/oral opening to different positions on the ventral side (one of the most basal acoel genera, Diopisthophorus, has a posteriorly positioned mouth/anus; Salvini-Plawen I978). The evolution of bilaterians with through gut (mouth + anus), which comprise all bilaterians except the acoelomorphs, the Platyhelminthes and Xenoturbella spp., was another key item in bilaterian evolution. According to van den Biggelaar and Dictus (2005), this might have occurred from cnidarian-like organisms in three different ways: ( $\mathrm{I}$ ) the blastopore maintained its posterior position becoming the anus, and a mouth developed later (Deuterostomia); (2) the posterior dorsal side of the blastopore extended (probably by proliferation as in some extant molluscs) shifting the mouth anteriorly towards the ventral side while the anus formed later (Protostomia); and (3) the body axis extended only along the dorsal side associated with the transformation of the blastopore into a longitudinal slit whose margins later fused in the middle, giving a tube with an anterior mouth opening and a posterior anal opening. This mode of blastopore closure, called amphistomy, has been proposed several times as a way to derive at a stroke the typical bilaterian body-plan features from a radial 
Gastraea from cnidarian adults (enterocoel theory of Sedgwick i 884), from benthic bilaterogastreas (Jägersten 1955) or from trochophora-type primary ciliary larvae (Arendt et al. 200 I). In the last case, the expression of otx and Bra was considered sufficient evidence to derive both mouth and anus from blastoporal regions. There is a general consensus, however, that primary larvae are not primitive but derived, not truly homologous, and prone to convergence (Sly et al. 2003). Moreover, Bra and otx, besides their clear roles in gastrulation and in specifying anterior body regions respectively, are also activated anew in any invagination movements (e.g. Bra in stomodeum formation) and in all sorts of ciliary bands (Otx); therefore their expression in larvae is probably due to convergence and needs to be reassessed. Further, in most embryos of molluscs, the blastopore does not contribute to the formation of the anus as required by the amphistomy concept (see van den Biggelaar and Dictus 2005 for references). Finally, according to the concept of amphistomy in its original formulation, head formation is expected at one side of the blastopore, and the opposite side should be posterior. Thus, the animal-vegetal axis of eggs and embryos which is parallel to the A-P axis now becomes parallel to the $\mathrm{D}-\mathrm{V}$ axis, whereas the A-P axis is made orthogonal to it. The main consequence is that the orientation of the expression domains of axial patterning genes is not in register between ancestor and descendant. Altogether, whereas amphistomic mechanisms may fit the specific developmental features of some lophotrochozoans (e.g. annelids) it cannot be extrapolated as a general mechanism, as in the original enterocoel theory (Sedgwick I 884) and variations thereof (Jägersten I955, Arendt et al. 200 I), to explain bilaterian evolution.

\section{CONCLUSIONS AND PROSPECTS}

New molecular phylogenies, in particular the proposal that acoelomorph flatworms are the earliest extant bilaterians, and the realisation that radial cnidarians have the axial features of bilaterians, are currently helping to unravel the sequential evolution of what once appeared to be a number of phylogenetically coincident character changes. Thus, key changes in bilaterian evolution are spread along several steps, which allow character states to be polarised. This argues against the complex Urbilateria hypothesis and helps us to see the evolution of the Bilateria as a series of successive Last Common Ancestor (LCA) nodes connected by stem ancestors along which new characters were acquired (Valentine 2006).

Refinements in data acquisition, evolution models, fossil record, molecular phylogenies, gene expression data (in particular forthcoming data on the 
expression of developmental genes in embryos and adults of acoelomorphs) and functional evo-devo will in the next few years be instrumental in unravelling the sequential evolution of clades at the base of the Deuterostomia, the Ecdysozoa and the Lophotrochozoa.

\section{ACKNOWLEDGEMENTS}

We thank Alessandro Minelli and Giuseppe Fusco for kindly inviting one of us (J.B.) to participate and deliver a talk at the Evo-Devo Meeting in Venice, from which this chapter is a summary. Constructive criticism, corrections and text editing by one reviewer are highly appreciated. These studies were supported by grants from the Generalitat de Catalunya and from CICYT (Ministerio de Ciencia y Tecnologia).

\section{REFERENCES}

Adoutte, A., Balavoine, G., Lartillot, N. \& de Rosa, R. 1999. Animal evolution: the end of intermediate taxa? Trends in Genetics I 5, 104-108.

Aguinaldo, A. M. A., Turbeville, J. M., Linford, L. S. et al. I 997. Evidence for a clade of nematodes, arthropods and other moulting animals. Nature 387, 489-493.

Arendt, D., Technau, U. \& Wittbrodt, J. 200 I. Evolution of the bilaterian larval foregut. Nature 409, 8I-85.

Ax, P. 1996. Multicellular Animals. A New Approach to the Phylogenetic Order in Nature, Vol. I. Berlin: Springer.

Baguñà, J. \& Riutort, M. 2004. The dawn of bilaterian animals: the case of the acoelomorph flatworms. BioEssays 26, 1046-1057.

Blair, J. E., Ikeo, K., Gojobori, T. \& Hedges, S. B. 2002. The evolutionary position of nematodes. BMC Evolutionary Biology 2, 7.

Brooke, N. M., García-Fernández, J. \& Holland, P. W. H. I 998. The ParaHox gene cluster is an evolutionary sister of the Hox cluster. Nature 392, 920-922.

Budd, G. E. 2003. The Cambrian fossil record and the origin of phyla. Integrative \& Comparative Biology 43, I 57-165.

Butterfield, N. J. 2006. Hooking some stem-group 'worms': fossil lophotrochozoans in the Burgess Shale. BioEssays 28, I I 6 I-I 66.

Carroll, S. B., Grenier, H. J. K. \& Weatherbee, S. D. 200 I. From DNA to Diversity. Malden: Blackwell Science.

Chourrout, D., Delsuc, F., Chourrout, P. et al. 2006. Minimal ProtoHox cluster inferred from bilaterian and cnidarian Hox complements. Nature 442, 684-687.

Conway Morris, S. 2006. Darwin's dilemma: the realities of the Cambrian 'explosion'. Philosophical Transactions of the Royal Society of London B $36 \mathbf{I}$, 1069-1083.

Cook, C. E., Jiménez, E., Akam, M. \& Saló, E. 2004. The Hox gene complement of acoel flatworms, a basal bilaterian clade. Evolution \& Development 6, I $54-163$. 
de Jong, D. M., Hislop, N. R., Hayward, D. C. et al. 2006. Components of both major axial patterning systems of the Bilateria are differentially expressed along the primary axis of a 'radiate' animal, the anthozoan cnidarian Acropora millepora. Developmental Biology 298, 632-643.

De Robertis, D. M. \& Sasai, Y. I996. A common plan for dorsoventral patterning in Bilateria. Nature 380, 37-40.

Donoghue, M. J. 2005. Key innovations, convergence, and success: macroevolutionary lessons from plant phylogeny. Paleobiology 31, 77-93.

Dopazo, H., Santoyo, J. \& Dopazo, J. 2004. Phylogenomics and the number of characters required for obtaining an accurate phylogeny of eukaryote model species. Bioinformatics 20 (supplement I), i I I 6-i I 2 I.

Erwin, D. H. \& Davidson, E. H. 2002. The last common bilaterian ancestor. Development I 29, $302 \mathrm{I}-3032$.

Extavour, C. G., Pang, K., Matus, D. Q. \& Martindale, M. Q. 2005. vasa and nanos expression patterns in a sea anemone and the evolution of bilaterian germ cell specification mechanisms. Evolution \& Development 7, $20 I-2 I 5$.

Finnerty, J. R. \& Martindale, M. Q. I 999. Ancient origins of axial patterning genes: Hox genes and ParaHox genes in the Cnidaria. Evolution \& Development $\mathbf{~}$, I 6-23.

Finnerty, J. R., Pang, K., Burton, P., Paulson, D. \& Martindale, M. Q. 2004. Origins of bilateral symmetry: Hox and $d p p$ expression in a sea anemone. Science 304, I335-1337.

Glenner, H., Hansen, A. J., Sorensen, M. V. et al. 2004. Bayesian inference of the Metazoan phylogeny: a combined molecular and morphological approach. Current Biology 14, I 644-1649.

Glinka, A., Wu, W., Onichtchouk, D., Blumenstock, C. \& Niehrs, C. I 997. Head induction by simultaneous repression of Bmp and Wnt signalling in Xenopus. Nature 389, 517-519.

Gould, S. J. I 989. Wonderful Life: The Burgess Shale and the Nature of History. New York: W.W. Norton.

Guder, C., Pinho, S., Nascak, T. G. et al. 2006. An ancient Wnt-Dickkopf antagonism in Hydra. Development I 33, 90 I-9 I I.

Halanych, K. M., Bachelor, J., Aguinaldo, A. M. A. et al. 1995. I $8 \mathrm{~S}$ rDNA evidence that lophophorates are protostome animals. Science 267, I64I-I 643.

Hayward, D. C., Samuel, G., Pontynen, P. C., Catmull, J. \& Saint, R. 2002. Localized expression of a dpp/BMP $2 / 4$ ortholog in a coral embryo. Proceedings of the National Academy of Sciences of the USA 99, 8106-8 I I I.

Holland, L. Z. 2000. Body-plan evolution in the Bilateria: early anteroposterior patterning and the deuterostome-protostome dichotomy. Current Opinion in Genetics \& Development 1 0, 434-442.

Holland, N. D. 2003. Early central nervous system evolution: an era of skin brains? Nature Reviews Neuroscience 4, I-I I.

Holland, P. W. H. 1998. Major transitions in animal evolution: a developmental genetic perspective. American Zoologist 38, 829-842. 
Hübner, C. 2006. Hox genes, homology and axis formation: The application of morphological concepts to evolutionary developmental biology. Theory in Biosciences. 1 24, 37 I-396.

Hyman, L. H. 195 I. The Invertebrates, Vol. 2. Platyhelminthes and Rhynchocoela. New York: McGraw-Hill.

Jägersten, G. I955. On the early phylogeny of the Metazoa. The bilaterogastrea theory. Zoologiska bidrag frain Uppsala 30, $321-354$.

Jeffroy, O., Brinkmann, H., Delsuc, F. \& Philippe, H. 2006. Phylogenomics: the beginning of incongruence? Trends in Genetics 22, 225-23 I.

Jenner, R. A. 2000 . Evolution of animal body plans: the role of metazoan phylogeny at the interface between pattern and process. Evolution \& Development 2, 208-22I.

Jiménez-Guri, E., Paps, J., García-Fernández, J. \& Saló, E. 2006. Hox and ParaHox genes in Nemertodermatida, a basal bilaterian clade. International Journal of Developmental Biology 50, 675-679.

Jondelius, U., Ruiz-Trillo, I., Baguñà, J. \& Riutort, M. 2002. The Nemertodermatida are basal bilaterians and not members of the Platyhelminthes. Zoologica Scripta 3I, 20I-2I5.

Kamm, K., Schierwater, B., Jakob, W., Dellaporta, S. L. \& Miller, D. J. 2006. Axial patterning and diversification in the Cnidaria predate the Hox system. Current Biology I 6, I-7.

Kimmel, C. B. 1996. Was Urbilateria segmented? Trends in Genetics I 2, 329-33I.

Lartillot, N., Brinkmann, H. \& Philippe, H. 2007. Suppression of long-branch attraction artefacts in the animal phylogeny using a site-heterogeneous model. BMC Evolutionary Biology 7, supplement I, $S_{4}$.

Lee, P. N., Pang, D., Matus, D. Q. \& Martindale, M. Q. 2006. A WNT of things to come: evolution of Wnt signalling and polarity in cnidarians. Seminars in Cell \& Developmental Biology 17, I 57-167.

Mallatt, J. \& Giribet, G. 2007. Further use of nearly complete $28 \mathrm{~S}$ and i $8 \mathrm{~S}$ rRNA genes to classify Ecdysozoa: 37 more arthropods and a kinorhynch. Molecular Phylogenetics and Evolution 40(3), 772-794.

Marshall, C. R. 2006. Explaining the Cambrian 'explosion' of animals. Annual Review of Earth \& Planetary Sciences 34, 355-384.

Martindale, M. Q. 2005. The evolution of metazoan axial properties. Nature Reviews Genetics 6, 917-927.

Martindale, M. Q., Pang, K. \& Finnerty, J. R. 2004. Investigating the origins of triploblasty: 'mesodermal' gene expression in a diploblastic animal, the sea anemone Nematostella vectensis (phylum, Cnidaria; class, Anthozoa). Development I 3 I, 2463-2474.

Matus, D. Q., Thomsen, G. H. \& Martindale, M. Q. 2006. Dorso/Ventral genes are asymmetrically expressed and involved in germ-layer demarcation during cnidarian gastrulation. Current Biology I6, 499-505.

Meinhardt, H. 2002. The radial-symmetric hydra and the evolution of the bilateral body plan: an old body became a young brain. BioEssays 24, I85-191. 
Nichols, S. A., Dirks, W., Pearse, J. S. \& King, N. 2006. Early evolution of animal cell signalling and adhesion genes. Proceedings of the National Academy of Sciences USA 103, I 245 I-I 2456.

Nielsen, C. \& Martinez, P. 2003. Patterns of gene expression: homology or homocracy? Development, Genes \& Evolution 213 , I $49-154$.

Pasquinelli, A. E., McCoy, A., Jiménez, E. et al. 2003. Expression of the 22 nucleotide let-7 heterochronic RNA throughout the Metazoa: a role in life history evolution? Evolution \& Development 5, 372-378.

Peterson, K. J. 2005. Macroevolutionary interplay between planktic larvae and benthic predators. Geology 33, 929-932.

Peterson, K. J., McPeek, M. A. \& Evans, D. A. D. 2005. Tempo and mode of early animal evolution: inferences from rocks, Hox, and molecular clocks. Paleobiology 3 I (supplement 2), 36-55.

Philippe, H., Delsuc, F., Brinkmann, H. \& Lartillot, N. 2005. Phylogenomics. Annual Review of Ecology, Evolution and Systematics 36, 541-562.

Philippe, H. \& Telford, M. J. 2006. Large-scale sequencing and the new animal phylogeny. Trends in Ecology \& Evolution 21, 61 4-620.

Rentzsch, F., Anton, R., Saina, M. et al. 2006. Asymmetric expression of the BMP antagonists chordin and gremlin in the sea anemone Nematostella vectensis: Implications for the evolution of axial patterning. Developmental Biology 296, 375-387.

Ruiz-Trillo, I., Paps, J., Loukota, M. et al. 2002. A phylogenetic analysis of myosin heavy chain type II sequences corroborates that Acoela and Nemertodermatida are basal bilaterians. Proceedings of the National Academy of Sciences of the USA 99, I I 246-I I 25 I.

Ruiz-Trillo, I., Riutort, M., Fourcade, H. M., Baguñà, J. \& Boore, J. L. 2004. Mitochondrial genome data support the basal position of Acoelomorpha and the polyphyly of the Platyhelminthes. Molecular Phylogenetics \& Evolution 33 , $32 \mathrm{I}-332$.

Ruiz-Trillo, I., Riutort, M., Littlewood, D. T. J., Herniou, E. A. \& Baguñà, J. I 999. Acoel flatworms: earliest extant bilaterian metazoans, not members of Platyhelminthes. Science 283, 1919-1923.

Salvini-Plawen, L. V. 1978. On the origin and evolution of the lower Metazoa. Zeitschrift für Zoologische Systematik und Evolutionsforschung I6, $40-88$.

Sedgwick, W. i 884. On the origin of metameric segmentation and some other morphological questions. Quarterly Journal of the Microscopical Society 24, 43-82.

Sempere, L., Cole, C. N., McPeek, M. A. \& Peterson, K. J. 2006. The phylogenetic distribution of Metazoan microRNAs: insights into evolutionary complexity and constraint. Journal of Experimental Zoology B (Molecular and Developmental Evolution) 306, 575-588.

Sly, B. J., Snoke, M. S. \& Raff, R. R. 2003. Who came first - larvae or adults? Origins of bilaterian metazoan larvae. International Journal of Developmental Biology 47, 623-632. 
Stark, A., Brennecke, J., Bushati, N., Russell, R. B. \& Cohen, S. M. 2005. Animal microRNAs confer robustness to gene expression and have a significant impact on $3^{\prime}$ UTR evolution. Cell I 23 , I-I 4 .

Stephenson, T. A. 1926. British Sea Anemones. London: The Ray Society.

Technau, U. 200I. Brachyury, the blastopore and the evolution of the mesoderm. BioEssays 23, 788-794.

Telford, M. J., Lockyer, A. E., Cartwright-Finch, C. \& Littlewood, D. T. J. 2003. Combined large and small subunit ribosomal RNA phylogenies support a basal position of the acoelomorph flatworms. Proceedings of the Royal Society of London B 270, 1077-1083.

Valentine, J. W. 2004. On the Origin of Phyla. Chicago: University of Chicago Press.

Valentine, J. W. 2006. Ancestors and urbilateria. Evolution \& Development 8, $39 \mathrm{I}-393$.

van den Biggelaar J. A. M. \& Dictus W. J. A. G. 2005. Gastrulation in the molluscan embryo. In C. Stern (ed.) Gastrulation, Cold Spring Harbor Laboratory Press. pp. $63-77$.

Wienholds, E. \& Plasterk, R. H. A. 2005. MicroRNA function in animal development. FEBS Letters 579, 591 I-5922.

Willmer, P. I990. Invertebrate Relationships: Patterns in Animal Evolution. Cambridge University Press: Cambridge. 